\title{
Multi-Band Cognitive Radio Spectrum Sensing for Quality-of-Service Traffic
}

\author{
Seung-Jun Kim, Guobing Li, and Georgios B. Giannakis
}

\begin{abstract}
Cognitive radios (CRs) are capable of sensing the RF spectrum to identify idle bands dynamically, and transmit opportunistically so as not to interfere with cohabiting primary users (PUs) over the same bands. In this work, spectrum sensing algorithms for CRs that support quality-of-service (QoS) traffic are investigated. Multiple bands are sensed in parallel to reduce the sensing delay, while ensuring a fixed minimum rate for CR transmissions with a given outage probability. Interference constraints are also imposed to protect PU transmissions. Both fixed sample size (FSS) as well as sequential sensing algorithms are developed to minimize the sensing delay. In the sequential sensing case, a bank of sequential probability ratio tests (SPRTs) are run in parallel to detect $P U$ presence in all bands concurrently. Notably, the parameters for the detectors can be obtained via convex optimization. Numerical tests demonstrate that sequential sensing yields average sensing delays significantly smaller than those of FSS sensing.
\end{abstract}

Index Terms-Cognitive radio, spectrum sensing, sequential probability ratio test, quality-of-service, cross-layer design.

\section{INTRODUCTION}

$\mathbf{T}$ HE cognitive radio (CR) concept is a promising idea that can alleviate the spectrum scarcity issue. CRs are endowed with the capability of sensing the RF environment so that they can opportunistically access those frequency bands not occupied by licensed primary users (PUs) in a given time and geographical area [14], [16]. Other tasks that enable CR spectrum sharing include spectrum access, transmitter-receiver handshake, and spectrum mobility management [1]. The focus of the present work is placed on spectrum sensing.

CR spectrum sensing, whose goal is to detect the presence of PU signals in the desired frequency bands, is often a challenging task due to the lack of cooperation from the PU systems. Moreover, when prior knowledge on the PU signal characteristics is not available, coherent demodulators such as matched filter detectors cannot be employed. Under a stringent interference requirement to protect the PU systems,

Manuscript received January 25, 2011; revised July 27, 2011; accepted August 3, 2011. The associate editor coordinating the review of this paper and approving it for publication was I.-M. Kim.

This work was supported by NSF grants CCF 0830480 and CON 014658, and by a QNRF-NPRP award 09-341-2-128. G. Li was supported by China Scholarship Council (CSC), "863" Program of China under Grant 2009AA011801 and NSFC under grant 60872028, 60832007 and 60902045. Part of this work was presented at the IEEE GLOBECOM Conference held in Miami, FL during December 6-10, 2010.

S.-J. Kim and G. B. Giannakis are with the Dept. of ECE, University of Minnesota, 200 Union Street SE, Minneapolis, MN 55455, U.S.A. (e-mail: \{seungjun, georgios\}@umn.edu).

$\mathrm{G}$. $\mathrm{Li}$ is with the School of Electronic and Information Engineering, Xi' an Jiaotong University, Xi'an, Shaanxi 710049, P.R.C. (e-mail: gbli@mail.xjtu.edu.cn). This work was performed during his visit to the University of Minnesota.

Digital Object Identifier 10.1109/TWC.2011.081611.110182 which translates to a tight miss detection probability bound, non-coherent detectors based on energy [3], or features such as cyclostationarity [15], may require a large number of measurements, and incur significant sensing delay.

Sensing delay has been recognized as an important factor that can affect the overall performance of CR systems. The sensing-throughput trade-off was studied in [12], motivated by the observation that prolonged sensing delay can reduce the time left for data transmission. A similar throughputrelated criterion was employed by [9] to design a multiband serial spectrum sensing algorithm. Needless to say, sensing delay is of paramount importance when the CR system conveys quality-of-service (QoS) traffic that has hard delay requirements. Delay analyses for QoS traffic were performed for cognitive radio networks in [19], [21]. On the other hand, when supporting QoS traffic, it is at least as important to provide a fixed data rate with low outage probability rather than maximize throughput [22].

To sustain the requested data rate with a low chance of outages under the PU-CR hierarchy, it is critical to sense a wide band of spectrum. A multi-band sensing architecture is a natural candidate to achieve this, and has been widely advocated in the CR literature [18], [20], [5], [24]. In order to reduce sensing delay to accommodate QoS traffic, it is desirable to sense the entire multi-band spectrum in parallel to find a set of unoccupied bands.

There are two possibilities in performing parallel multiband sensing. One is to fix the number of samples taken per band a priori, which is henceforth referred to as fixed sample size (FSS) sensing. The other is to perform sequential detection per band, in which case, the sample sizes can vary depending on the sample realizations. The premise of sequential detection is improved sensing delay on the average. FSS sensing has been analyzed in [18] with the objective of maximizing CR throughput. Likewise, sequential sensing algorithms were investigated in [4] and [23] as well as in [11] using dynamic programming to also maximize throughput.

In this work, both FSS and sequential sensing algorithms are developed to minimize the multi-band sensing delay while supporting a fixed minimum rate with a given outage probability bound. Interference constraints to protect PU systems are also imposed. For FSS sensing, a series of convex feasibility problems is formulated to obtain optimal detector parameters. In the case of sequential sensing, an architecture of running sequential probability ratio tests (SPRTs) in parallel is adopted. SPRT is an online detector applicable to simple binary hypothesis testing problems with widely appreciated merits [7], [17]. However, tackling the multi-band sensing problem with the 
parallel SPRT architecture entails a unique challenge due to random stopping times of the tests. This is mitigated here by a convex optimization formulation.

The rest of the paper is organized as follows. Sec. II provides the system model. Sec. III first models the interference and outage probability constraints for FSS sensing, and introduces a sample size minimization problem that can be solved via convex optimization tools. Sec. IV deals with sequential sensing. Numerical tests are presented in Sec. V, followed by conclusions in Sec. VI.

\section{System Model}

Consider a CR network opportunistically sharing the spectrum with $J$ licensed PUs. Each CR is equipped with a multi-band transceiver through which it can communicate over $M$ orthogonal narrowband channels using such techniques as orthogonal frequency division multiplexing (OFDM). As is often the case with practical implementation, a half-duplex constraint is present, i.e., the CR transceiver cannot transmit in one band while listening in other bands. It is assumed that the spectrum occupancy of the PUs is independent across bands. The goal of CR spectrum sensing is to detect the PU occupancy on the individual bands.

To this end, binary hypothesis testing can be performed per band $m \in\{1,2, \ldots, M\}$ in order to decide presence or absence of the PU signal. Let $r_{n}^{(m)}$ denote the signal sample received by the CR under consideration on band $m$ at the $n$-th sampling instant. Then, the two hypotheses $\mathcal{H}_{0}^{(m)}$ and $\mathcal{H}_{1}^{(m)}$ on band $m$, which correspond to the absence and presence of PU activity on that band, respectively, can be represented as

$$
\begin{cases}\mathcal{H}_{0}^{(m)}: & r_{n}^{(m)}=z_{n}^{(m)} \\ \mathcal{H}_{1}^{(m)}: & r_{n}^{(m)}=h_{n}^{(m)} s_{n}^{(m)}+z_{n}^{(m)}\end{cases}
$$

where $h_{n}^{(m)}$ is the complex channel coefficient, $s_{n}^{(m)}$ the PU signal sample, and $z_{n}^{(m)}$ the independent and identically distributed (i.i.d.) additive white complex Gaussian noise sample with zero mean and variance $\sigma^{2}$, all on band $m$ at time $n$. The PU signal $s_{n}^{(m)}$ is assumed to be i.i.d. Gaussian with mean zero and unit variance for all $m$. (The latter is without loss of generality since the known channel gain can be otherwise properly scaled to account for non-unit variance.) It is also assumed that the channels between the PUs and the CR are quasi-static; that is, for all $m$, the channel coefficients $\left\{h_{n}^{(m)}\right\}$ stay invariant during the detection process. Let also $G^{(m)}:=\left|h_{n}^{(m)}\right|^{2}>0$ denote the channel gain on band $m$, and $\pi_{0}^{(m)}$ and $\pi_{1}^{(m)}$ the prior probabilities of the two hypotheses $\operatorname{Pr}\left\{\mathcal{H}_{0}^{(m)}\right\}$ and $\operatorname{Pr}\left\{\mathcal{H}_{1}^{(m)}\right\}$, respectively. The conditional p.d.f.'s of $r_{n}^{(m)}$ under $\mathcal{H}_{0}^{(m)}$ and $\mathcal{H}_{1}^{(m)}$ are denoted by $p_{0}^{(m)}\left(r_{n}^{(m)}\right)$ and $p_{1}^{(m)}\left(r_{n}^{(m)}\right)$, respectively.

Remark 1. The PU-to-CR channel knowledge $\left\{G^{(m)}\right\}$ can be obtained during the acquisition stage, where the CR receiver can learn the channels blindly, or, by overhearing the pilots transmitted by the PU system. Especially for immobile transceivers, a sufficient number of samples can be used in this stage to ensure practically error-free channel estimates. That is, the goal here is to obtain reliable channel gain estimates, possibly accounting for dynamic changes in PU occupancy, using a long observation window. Based on these acquired channel estimates, the algorithms of the present work are intended for the operational stage to detect PU presence even employing energy detection, while incurring minimum sensing delay. A justification of PU-to-CR channel knowledge in the context of digital TV systems is provided in [18], although the issue of sensing delay may be less prominent there due to quasi-static PU occupancy.

Under this model, the minimum error probability detector for (1) amounts to a likelihood ratio test, which here reduces to an energy detector [10, Ch. 5]. Specifically, based on $N$ samples $\left\{r_{n}^{(m)}\right\}_{n=1}^{N}$ per band, the test statistic $t_{N}^{(m)}$ can be expressed as

$$
t_{N}^{(m)}=\sum_{n=1}^{N}\left|r_{n}^{(m)}\right|^{2}
$$

which is related to the log-likelihood ratio $\Lambda_{N}^{(m)} \triangleq$ $\sum_{n=1}^{N} \log \frac{p_{1}^{(m)}\left(r_{n}^{(m)}\right)}{p_{0}^{(m)}\left(r_{n}^{(m)}\right)}$ through

$\Lambda_{N}^{(m)}=\frac{G^{(m)}}{\left(G^{(m)}+\sigma^{2}\right) \sigma^{2}} t_{N}^{(m)}+N \log \sigma^{2}-N \log \left(G^{(m)}+\sigma^{2}\right)$.

Two approaches are available for testing the binary hypotheses in (1): FSS and sequential tests. An FSS test collects a pre-determined number, $N$, of samples before declaring that either of the two hypotheses is in force. A sequential test stops as soon as enough confidence has been gained as to which of the hypotheses is true. For this reason, sequential tests have the potential to reduce the detection delay on the average [7]. Therefore, sequential tests are of particular interest for CRs when supporting delay-limited traffic where the sensing delay contributes to the overall packet delay.

On the other hand, QoS traffic typically comes with strict minimum rate requirements, and an outage will occur if the minimum rate cannot be supported. To account for these issues, optimal FSS tests are developed in the ensuing section. Sequential schemes are investigated in Sec. IV. Note that collaboration in sensing is not considered, and the sensing delay analyzed in this contribution does not account for the protocol issues such as control signaling.

\section{FiXed S Ample Size Sensing}

In this section, we formulate a constrained optimization problem to find the minimum sample size for FSS sensing with a guaranteed outage probability bound as well as strict interference constraints to protect the PU transmissions.

\section{A. Interference Constraints}

By invoking the central limit theorem for $N$ sufficiently large as in [12], it follows readily that the test statistic in (2) is asymptotically Gaussian distributed under each hypothesis; that is,

$t_{N}^{(m)} \sim \begin{cases}\mathcal{N}\left(N \sigma^{2}, N \sigma^{4}\right) & \text { under } \mathcal{H}_{0}^{(m)} \\ \mathcal{N}\left(N\left(\sigma^{2}+G^{(m)}\right), N\left(\sigma^{2}+G^{(m)}\right)^{2}\right) & \text { under } \mathcal{H}_{1}^{(m)}\end{cases}$ 
where $\mathcal{N}\left(\mu, v^{2}\right)$ denotes the real-valued Gaussian distribution with mean $\mu$ and variance $v^{2}$.

Using (4), one can express the false alarm probability $\alpha_{N}^{(m)}$ and the miss detection probability $\beta_{N}^{(m)}$, for a given threshold $\gamma_{N}^{(m)}$ of the FSS energy detector on band $m$, as

$$
\begin{aligned}
\alpha_{N}^{(m)} & =\operatorname{Pr}\left\{t_{N}^{(m)}>\gamma_{N}^{(m)} \mid \mathcal{H}_{0}^{(m)}\right\}=Q\left(\frac{\gamma_{N}^{(m)}-N \sigma^{2}}{\sigma^{2} \sqrt{N}}\right) \\
\beta_{N}^{(m)} & =\operatorname{Pr}\left\{t_{N}^{(m)}<\gamma_{N}^{(m)} \mid \mathcal{H}_{1}^{(m)}\right\} \\
& =Q\left(\frac{N\left(\sigma^{2}+G^{(m)}\right)-\gamma_{N}^{(m)}}{\left(\sigma^{2}+G^{(m)}\right) \sqrt{N}}\right)
\end{aligned}
$$

where $Q(x) \triangleq \frac{1}{\sqrt{2 \pi}} \int_{x}^{\infty} e^{-t^{2} / 2} d t$.

Using the definition of $Q(x)$, simple inspection of (5) and (6) reveals that for $N$ fixed, $\alpha_{N}^{(m)}$ decreases while $\beta_{N}^{(m)}$ increases with $\gamma_{N}^{(m)}$ increasing. The resulting trade-off curve can be traversed by adjusting $\gamma_{N}^{(m)}$. Moreover, with $\gamma_{N}^{(m)}=\gamma^{(m)}$ fixed, increasing $N$ lowers both $\alpha_{N}^{(m)}$ and $\beta_{N}^{(m)}$.

In the context of $\mathrm{CR}$ sensing, miss detection has to be strictly regulated in order to control CR interference to the PUs whose presence is overlooked. False alarms are also undesirable because they result in under-utilization of the available bands for $\mathrm{CR}$ data transmission. These considerations justify per-band constraints on the false alarm and the miss detection rates, as

$$
\begin{aligned}
\alpha_{N}^{(m)} \leq \bar{\alpha}^{(m)}, & m=1,2, \ldots, M \\
\beta_{N}^{(m)} \leq \bar{\beta}^{(m)}, & m=1,2, \ldots, M
\end{aligned}
$$

where $\bar{\alpha}^{(m)}$ and $\bar{\beta}^{(m)}$ are pre-specified bounds.

In addition, if one knows a priori the set of bands that PUs will occupy, it is sensible to constrain the interference on a per-PU basis [18]. For example, when a PU occupies a wide band, and strong error correction coding is employed across the band, the PU signal may be resilient to partialband interference. In such a case, it would be reasonable to constraint an interference probability averaged over the band. With a total of $J(<M)$ PUs present, let $S_{j}$ denote the set of bands that PU $j$ occupies for $j=1,2, \ldots, J$, where $\cup_{j} S_{j}=$ $\{1,2, \ldots, M\}$. Then, for a set of nonnegative weights $\left\{w_{j m}\right\}$ with $\sum_{m \in S_{j}} w_{j m}=1$, pertinent interference constraints take the form

$$
\sum_{m \in S_{j}} w_{j m} \beta_{N}^{(m)} \leq \bar{\beta}_{j}, \quad j=1, \ldots, J .
$$

Note that the weights are related to relative importance of each band to the PU transmissions. For instance, PU $j$ that occupies the set of bands $S_{j}$ may find certain bands in $S_{j}$ more susceptible to interference than other bands, e.g., because they are used for control signaling or pilot transmissions.

At this point, it is useful to remember that constraints (7), (8) and (9) are all expressible in terms of the detection thresholds $\left\{\gamma_{N}^{(m)}\right\}_{m=1}^{M}$, the choice of which will be optimized in Sec. III-D.

\section{B. Outage Probability Constraints}

Delay-limited traffic often entails a hard minimum rate requirement. When the minimum rate cannot be achieved, an outage occurs. Thus, it is desirable to regulate the outage probability. Note that such design consideration is quite different from that of average throughput maximization pursued in [9], [11], [12], [18], where supporting QoS traffic is not of main concern.

Suppose that band $m$ can support a CR rate of $R_{0}^{(m)}$ if no PU is present. Rates $\left\{R_{0}^{(m)}\right\}$ are deterministically known and supportable because the channel gains of the CR links are assumed non-random and fixed. Such information can be initially learned during link establishment, and updated during data transmission using pilot signals. With $\mathrm{PU}(\mathrm{s})$ possibly present, the rate supported on band $m$ can be modeled as a random variable

$$
R^{(m)} \triangleq R_{0}^{(m)} \mathbb{1}_{\left\{\mathcal{H}_{0}^{(m)} \text { true and } \mathcal{H}_{0}^{(m)} \text { correctly detected }\right\}}
$$

where $\mathbb{1}_{A}$ is the indicator function of the event $A$. Note that the underlying assumption is that if a PU is active on band $m$, and a CR transmitter sends a packet over the band owing to miss detection, the $\mathrm{CR}$ receiver cannot decode the message due to, e.g., interference. This is a conservative way of estimating the achievable rate of the CR system. The event of accepting $\mathcal{H}_{0}^{(m)}$ amounts to having $t_{N}^{(m)}<\gamma_{N}^{(m)}$ in the context of the FSS test. The random variable $R^{(m)}$ takes the value $R_{0}^{(m)}$ with probability $\pi_{0}^{(m)}\left(1-\alpha_{N}^{(m)}\right)$, and the value 0 with probability $1-\pi_{0}^{(m)}\left(1-\alpha_{N}^{(m)}\right)$. Its mean is clearly given by $\mathbb{E}\left\{R^{(m)}\right\}=$ $R_{0}^{(m)} \pi_{0}^{(m)}\left(1-\alpha_{N}^{(m)}\right)$.

A prime example of QoS traffic is the one that periodically generates a certain amount of traffic $R_{\min }$ to be delivered with small delay. An outage is declared if the total rate $R_{M} \triangleq$ $\sum_{m=1}^{M} R^{(m)}$ over all $M$ bands falls below the minimum rate target $R_{\min }$. Thus, the outage probability constraint becomes

$$
\operatorname{Pr}\left\{R_{M}<\mathrm{R}_{\min }\right\} \leq \epsilon
$$

where $\epsilon>0$ is a small constant prescribing the outage upper bound.

1) An Upper Bound to Outage Probability: The outage probability in (11) is hard to express in closed form as a function of detection parameters. For analytical tractability, Hoeffding's inequality [8] can be invoked to obtain an upper bound to this outage probability. The resulting upper bound can then be constrained not to exceed $\epsilon$ for a conservative design

$$
\operatorname{Pr}\left\{R_{M}<\mathrm{R}_{\min }\right\} \leq \exp \left[\frac{-2\left(\mathbb{E}\left\{R_{M}\right\}-\mathrm{R}_{\min }\right)^{2}}{\sum_{m=1}^{M} R_{0}^{(m)^{2}}}\right] \leq \epsilon .
$$

Under the typically satisfied assumption that $\mathrm{R}_{\min }<\mathbb{E}\left\{R_{M}\right\}$, the second inequality in (12) can be written as

$$
\mathbb{E}\left\{R_{M}\right\} \geq \mathrm{R}_{\min }+\sqrt{-\frac{\log \epsilon}{2} \sum_{m=1}^{M} R_{0}^{(m)^{2}}}
$$

It is now clear that the outage constraint has been translated to a constraint on having the average rate stay above $R_{\min }$ by a margin that is dependent on the outage target $\epsilon$. Let 
$\bar{R} \triangleq \sum_{m=1}^{M} R_{0}^{(m)} \pi_{0}^{(m)}$ denote the average achievable rate when sensing is free of false alarms. Since $\mathbb{E}\left\{R^{(m)}\right\}=$ $R_{0}^{(m)} \pi_{0}^{(m)}\left(1-\alpha_{N}^{(m)}\right)$, it is possible to re-write (13) as

$$
\sum_{m=1}^{M} R_{0}^{(m)} \pi_{0}^{(m)} \alpha_{N}^{(m)} \leq C_{1}(\epsilon)
$$

where

$$
C_{1}(\epsilon) \triangleq \bar{R}-\mathrm{R}_{\min }-\sqrt{-\frac{\log \epsilon}{2} \sum_{m=1}^{M} R_{0}^{(m)^{2}}} .
$$

Thus, the conservative outage constraint upper-bounds a weighted sum of the false alarm probabilities, which are expressible in terms of $\left\{\gamma_{N}^{(m)}\right\}_{m=1}^{M}$ (cf. (5)). Note also that since $C_{1}(\epsilon)$ must be positive, the feasible values that the pair $\left(\epsilon, \mathrm{R}_{\min }\right)$ can take are constrained. Specifically, $\epsilon$ is lowerbounded by

$$
\epsilon>\exp \left[-\frac{2\left(\bar{R}-\mathrm{R}_{\min }\right)^{2}}{\sum_{m=1}^{M} R_{0}^{(m)^{2}}}\right] .
$$

2) An Alternative Approximation: The outage upper bound in (12) often turns out to be quite loose, as will be exemplified by numerical tests in Sec. V. This motivates the goal of this subsection to obtain an alternative approximation to the outage probability, using once again the central limit theorem.

Lemma 1. Under mild regularity conditions, it holds that

$$
\frac{R_{M}-\mathbb{E}\left\{R_{M}\right\}}{\sqrt{\operatorname{var}\left\{R_{M}\right\}}} \underset{M \rightarrow \infty}{\sim} \mathcal{N}(0,1) .
$$

For uniformly bounded $\left\{R^{(m)}\right\}$, having $\operatorname{var}\left\{R_{M}\right\} \rightarrow \infty$ as $M \rightarrow \infty$ is sufficient for the central limit theorem to hold [6, p. 264]. This regularity condition is easily justifiable in practice. Taking $M$ large enough ${ }^{1}$, the random variable $R_{M}$ can thus be approximated as Gaussian with mean and variance given by

$$
\begin{aligned}
\mathbb{E}\left\{R_{M}\right\} & =\sum_{m=1}^{M} R_{0}^{(m)} \pi_{0}^{(m)}\left(1-\alpha_{N}^{(m)}\right) \\
\operatorname{var}\left\{R_{M}\right\} & =\sum_{m=1}^{M} R_{0}^{(m)^{2}}\left[\pi_{0}^{(m)}\left(1-\alpha_{N}^{(m)}\right)-\pi_{0}^{(m)^{2}}\left(1-\alpha_{N}^{(m)}\right)^{2}\right] .
\end{aligned}
$$

Therefore, the outage constraint (11) can be approximated as

$$
Q\left(\frac{\mathbb{E}\left\{R_{M}\right\}-\mathrm{R}_{\text {min }}}{\sqrt{\operatorname{var}\left\{R_{M}\right\}}}\right) \leq \epsilon
$$

which can be readily shown to be non-convex w.r.t. $\left\{\gamma_{N}^{(m)}\right\}_{m=1}^{M}$. To bypass this difficulty, a conservative approach

\footnotetext{
${ }^{1}$ Since the number of bands can only be finite, Lemma 1 merely provides an insight to facilitate tractable design, which must be verified by numerical tests. On the other hand, the idea of pursuing a multi-band CR architecture is to capitalize on "frequency diversity." That is, provided that $M$ is large, it will be likely that there are enough idle bands for the CRs to use. Modern wideband systems often use OFDM with the number of bands easily reaching 100 's. In Sec. V, numerical tests verifies our design with $M=10$ bands.
}

is taken once more by upper-bounding the variance of $R_{M}$. To this end, consider the following bound [cf. (18)]:

$$
\begin{aligned}
& {\left[\pi_{0}^{(m)}\left(1-\alpha_{N}^{(m)}\right)-\pi_{0}^{(m)^{2}}\left(1-\alpha_{N}^{(m)}\right)^{2}\right]} \\
& \leq f\left(\pi_{0}^{(m)}\right) \triangleq \begin{cases}\pi_{0}^{(m)}-\pi_{0}^{(m)^{2}} & \text { if } 0 \leq \pi_{0}^{(m)}<\frac{1}{2} \\
\frac{1}{4} & \text { if } \frac{1}{2} \leq \pi_{0}^{(m)} \leq 1\end{cases}
\end{aligned}
$$

which is obtained simply by viewing the l.h.s. of (20) as a quadratic function of $x:=\pi_{0}^{(m)}\left(1-\alpha_{N}^{(m)}\right)$, where $0 \leq x \leq$ $\pi_{0}^{(m)}$. Now, a conservative approximate outage constraint is given by

$$
Q\left(\frac{\mathbb{E}\left\{R_{M}\right\}-\mathrm{R}_{\min }}{\sqrt{\sum_{m=1}^{M} R_{0}^{(m)^{2}} f\left(\pi_{0}^{(m)}\right)}}\right) \leq \epsilon
$$

which is equivalent to

$$
\sum_{m=1}^{M} R_{0}^{(m)} \pi_{0}^{(m)} \alpha_{N}^{(m)} \leq C_{2}(\epsilon)
$$

where

$$
C_{2}(\epsilon) \triangleq \bar{R}-\mathrm{R}_{\min }-Q^{-1}(\epsilon) \sqrt{\sum_{m=1}^{M} R_{0}^{(m)^{2}} f\left(\pi_{0}^{(m)}\right)} .
$$

Again, $C_{2}(\epsilon)>0$ is necessary for feasibility.

\section{Optimization Problem}

The optimization problem of interest is to minimize the number of samples (and thus sensing delay) under the interference and outage constraints. The sensing delays of all bands are aligned due to a half-duplex hardware constraint. That is, a multi-band transceiver often cannot transmit in one band while sensing in another band. This makes it optimal to equalize the sensing durations over the bands, since finishing sensing on one band earlier than other bands simply translates to lower detection performance on that band without any benefit in rate. Specifically, consider the following optimization problem:

(P1)

$$
\min _{N \in \mathcal{N},\left\{\gamma_{N}^{(m)}\right\}} N
$$

$$
\begin{gathered}
\text { subject to } 0 \leq \alpha_{N}^{(m)} \leq \bar{\alpha}^{(m)}, \quad m=1, \ldots, M \\
0 \leq \beta_{N}^{(m)} \leq \bar{\beta}^{(m)}, \quad m=1, \ldots, M \\
\sum_{m \in S_{j}} w_{j m} \beta_{N}^{(m)} \leq \bar{\beta}_{j}, \quad j=1, \ldots, J \\
\sum_{m=1}^{M} R_{0}^{(m)} \pi_{0}^{(m)} \alpha_{N}^{(m)} \leq C(\epsilon) .
\end{gathered}
$$

Here, $\mathcal{N} \triangleq\left\{N_{\min }, N_{\min }+1, \ldots, N_{\max }\right\}$ with $N_{\min }$ and $N_{\max }$ denoting pre-selected minimum and maximum allowed sample sizes, respectively.

The constraints in (24) regulate the false alarm probabilities on each band. Constraints (25)-(26) limit the probability of interfering with the PUs due to miss detection: (25) constrains the miss detection per band, while (26) protects $J$ PUs that are known to occupy the set of bands $S_{1}, S_{2}, \ldots, S_{J}$. Constraint (27) is the rate outage constraint given the outage probability bound $\epsilon$, where $C(\epsilon)$ can be set equal to either $C_{1}(\epsilon)$ in (15), or, $C_{2}(\epsilon)$ in (23). 


\section{Optimal FSS Sensing}

Problem (P1) in Sec. III-C can be solved by searching for the minimum $N \in \mathcal{N}$, which renders the constraints (24)(27) feasible. For a given $N$, it is straightforward to check the feasibility of (24) and (25) using (5) and (6). Once they turn out to be feasible for a given $N$, the following feasibility problem can be solved to check whether the constraints (24)(27) are jointly satisfied:

$$
\min _{s,\left\{\gamma_{N}^{(m)}\right\}} s
$$

subject to (24) and (25)

$$
\begin{aligned}
& \sum_{m \in S_{j}} w_{j m} \beta_{N}^{(m)} \leq \bar{\beta}_{j} s, \quad j=1, \ldots, J \\
& \sum_{m=1}^{M} R_{0}^{(m)} \pi_{0}^{(m)} \alpha_{N}^{(m)} \leq C(\epsilon) s .
\end{aligned}
$$

Note that this problem is always feasible provided the constraints (24) and (25) are feasible, since the 1.h.s.'s of (29) and (30) are bounded. Moreover, if the optimal $s$ turns out to be less than or equal to 1 , then it can be deduced that the original constraints (24)-(27) are feasible for the considered $N$. To solve (P2), the following result is instrumental.

Proposition 1. Problem (P2) is a convex optimization problem when $\bar{\alpha}^{(m)} \leq \frac{1}{2}$ and $\bar{\beta}^{(m)} \leq \frac{1}{2}$, and thus can be efficiently solved.

Proof: The convexity follows from the fact that $Q(x)$ is convex when $Q(x) \leq \frac{1}{2}$, which implies that $\alpha_{N}^{(m)}$ and $\beta_{N}^{(m)}$, in (5) and (6), respectively, are convex w.r.t. $\gamma_{N}^{(m)}$. It is then straightforward to see that the feasible set defined by (28)(30) is convex.

Standard convex problems such as (P2) can be solved efficiently using, e.g., the interior point method [2]. Moreover, thanks to the monotonicity of $\alpha_{N}^{(m)}$ and $\beta_{N}^{(m)}$ w.r.t. $N$, the optimal $N$ can be found via binary search which requires solving (P2) at most $\left\lceil\log _{2}\left(N_{\max }-N_{\min }+1\right)\right\rceil$ times.

Once the optimal detector parameters have been obtained, FSS sensing gives PU occupancy decisions on all bands with fixed sensing delay. However, since $N$ has to be determined without observing actual sample realizations, even the optimized $N$ may be quite large, especially when the SNR is relatively low. The sequential sensing algorithm developed next can achieve significant savings in average sensing delay by allowing the sample size to depend on sample realizations.

Remark 2. The proposed approach will be most suitable for stationary or slowly moving PUs and CRs. In such a case, the channel gains and supported rates can be accurately estimated; see also Remark 1. The prior probabilities for PU activities can also be estimated and continuously updated similarly, an assumption often made in CR literature [24], [5]. The minimum required rate and the outage target can be deduced from application requirements, and are often assumed to be known [22], [21]. As long as channel statistics and rate requirements vary slowly, (P1) needs to be solved only infrequently, and few iterations will track optimal parameters. Overall, while the practicality of the proposed approach de- pends on actual deployment and application contexts, there are ample scenarios to which the proposed design can be applied.

\section{Sequential Sensing}

In this section, a sequential detection scheme is developed for multi-band spectrum sensing using a bank of SPRTs, one per band. The SPRT has a very simple structure where the likelihood ratio of the observed samples is tested against two thresholds. This test is known to minimize the average sample number (ASN) among all detectors that achieve the given false alarm and miss detection probabilities [7]. As QoS traffic benefits from minimal sensing delay, this property makes it particularly relevant to investigate the proposed architecture from both performance and complexity perspectives.

A key challenge associated with the proposed online multiband detector, however, is that parallel SPRTs do not yield the same sample sizes, simply because the latter are random variables which depend on the observed samples. As a result, the overall sensing delay will be dictated by the largest detection delay among those of the parallel detectors, until the set of bands that can support the requested rate is discovered. A rigorous design of sequential sensing based on dynamic programming was treated in [11] for maximizing average throughput.

The aim here is to retain the parallel SPRT structure for simplicity of implementation, while optimizing the SPRT parameters to mitigate the loss in delay performance due to disparate sample sizes. Common to the previous section, the objective is to minimize the sensing delay under an outage constraint on the supported rate as well as the interference constraints imposed to abide by the PU-CR hierarchy.

\section{A. Optimization Problem}

In SPRT, there are two thresholds against which the likelihood ratio is compared at each sampling time [7]. If the likelihood ratio exceeds either one of the thresholds, the detection process terminates and a decision is declared. Denote the two thresholds used for the SPRT on band $m$ as $A^{(m)}$ and $B^{(m)}$, where $A^{(m)}>B^{(m)}>0$ holds. Then, the SPRT on band $m$ operates at each sampling instant $n$ as follows:

$$
\begin{cases}\Lambda_{n}^{(m)} \geq \log A^{(m)} & \Rightarrow \text { choose } \mathcal{H}_{1}^{(m)} \\ \Lambda_{n}^{(m)} \leq \log B^{(m)} & \Rightarrow \text { choose } \mathcal{H}_{0}^{(m)} \\ \log B^{(m)}<\Lambda_{n}^{(m)}<\log A^{(m)} & \Rightarrow \text { take next sample }\end{cases}
$$

where the thresholds are related to the false alarm probability $\alpha^{(m)}$ and the miss detection probability $\beta^{(m)}$ as [7, p. 20]

$$
A^{(m)} \approx \frac{1-\beta^{(m)}}{\alpha^{(m)}} \text { and } B^{(m)} \approx \frac{\beta^{(m)}}{1-\alpha^{(m)}} .
$$

The ASN for band $m$ under $\mathcal{H}_{0}^{(m)}$, which is denoted by $\operatorname{ASN}_{0}^{(m)}$, for prescribed values of $\alpha^{(m)}$ and $\beta^{(m)}$ is given approximately by $[7$, p. 30]

$$
\begin{aligned}
\operatorname{ASN}_{0}^{(m)}\left(\alpha^{(m)}, \beta^{(m)}\right) \approx & \frac{1}{\mathcal{D}\left(p_{0}^{(m)} \| p_{1}^{(m)}\right)}\left[\alpha^{(m)} \log \frac{\alpha^{(m)}}{1-\beta^{(m)}}\right. \\
& \left.+\left(1-\alpha^{(m)}\right) \log \frac{1-\alpha^{(m)}}{\beta^{(m)}}\right]
\end{aligned}
$$


where $\mathcal{D}\left(p_{0}^{(m)} \| p_{1}^{(m)}\right)$ is the Kullback-Leibler divergence of the two p.d.f.'s $p_{0}^{(m)}$ and $p_{1}^{(m)}$. In the present context, the latter is equal to $\mathcal{D}\left(p_{0}^{(m)} \| p_{1}^{(m)}\right)=\log \left(1+\mathrm{SNR}^{(m)}\right)-\frac{\mathrm{SNR}^{(m)}}{1+\mathrm{SNR}^{(m)}}$ with $\mathrm{SNR}^{(m)} \triangleq G^{(m)} / \sigma^{2}$; cf. (1). ${ }^{2}$

The proposed optimization problem is to minimize the maximum $\mathrm{ASN}_{0}^{(m)}$ over all bands $m \in\{1,2, \ldots, M\}$ subject to the interference and outage constraints:

$$
\begin{gathered}
\min _{\left\{\alpha^{(m)}\right\},\left\{\beta^{(m)}\right\}} \max _{m \in\{1, \ldots, M\}} \mathrm{ASN}_{0}^{(m)} \\
\text { subject to } 0 \leq \alpha^{(m)} \leq \bar{\alpha}^{(m)}, \quad m=1, \ldots, M \\
0 \leq \beta^{(m)} \leq \bar{\beta}^{(m)}, \quad m=1, \ldots, M \\
\sum_{m \in S_{j}} w_{j m} \beta^{(m)} \leq \bar{\beta}_{j}, \quad j=1, \ldots, J \\
\sum_{m=1}^{M} R_{0}^{(m)} \pi_{0}^{(m)} \alpha^{(m)} \leq C(\epsilon) .
\end{gathered}
$$

The per-band false alarm constraints in (34) are analogous to those in (24). Likewise, constraints (35)-(36) are interference constraints similar to those in (25)-(26). In the same way, (37) is the approximate outage constraint, whose justification is completely analogous to the FSS case detailed in Sections III-B1 and III-B2. The constant $C(\epsilon)$ can be chosen to be either $C_{1}(\epsilon)$ or $C_{2}(\epsilon)$.

Contrary to the case of (P2) for FSS sensing, the optimization variables in (P3) are the false alarm and the miss detection probabilities in each band. More interestingly, and to much practical appeal, the optimization for sequential sensing is seen to be simpler than that for the FSS counterpart, as one does not need to solve optimization problems for different sample sizes. Rather, the optimal $\left\{\alpha^{(m)}\right\}$ and $\left\{\beta^{(m)}\right\}$ determine the ASNs automatically.

The min-max formulation (P3) essentially renders the ASNs in different bands identical and minimal. The rationale behind this approach is that by aligning the ASNs, one can expect that the actual stopping times of the SPRTs are also not too distant from one another. Recall that the actual sensing delay is the time until a set of unoccupied bands is found enabling a combined rate above $R_{\min }$. Since one does not know a priori what will be such a set, it is reasonable to adopt a conservative approach that regards all bands as candidates, and attempts to minimize the stopping times of all bands. Moreover, (P3) can be efficiently solved for the reason stated in the following proposition.

\section{Proposition 2. Problem (P3) is a convex optimization prob- lem.}

Proof: The constraints are linear and thus convex. To show that the objective function is also convex, it is first shown that the function $\operatorname{ASN}_{0}^{(m)}(\alpha, \beta)$ is convex w.r.t. $\alpha$ and $\beta$, where

\footnotetext{
${ }^{2}$ The ASN under $\mathcal{H}_{1}^{(m)}$ is not relevant because the sensing procedure stops when enough idle bands to support $R_{\min }$ are identified; cf. Sec. IV-B. Thus, provided that channels are not in outage (i.e., there are actually enough free bands to support CR traffic in the first place), the overall sensing delay is influenced only by the ASNs under $\mathcal{H}_{0}^{(m)}$. In the rare case of outage, the sensing procedure will be eventually terminated if the delay exceeds a tolerable level. Thus, we do not propose to minimize the sensing delay in outage.
}

$\alpha, \beta \in(0,1)$. Clearly, $\operatorname{ASN}_{0}^{(m)}(\alpha, \beta)$ is twice differentiable, and the Hessian matrix is given by

$$
\frac{1}{\mathcal{D}\left(p_{0}^{(m)} \| p_{1}^{(m)}\right)}\left[\begin{array}{cc}
\frac{1}{\alpha}+\frac{1}{1-\alpha} & \frac{1}{1-\beta}+\frac{1}{\beta} \\
\frac{1}{1-\beta}+\frac{1}{\beta} & \frac{\alpha}{\beta^{2}}+\frac{1-\alpha}{(1-\beta)^{2}}
\end{array}\right] .
$$

Next, we argue that the positive $\mathcal{D}\left(p_{0}^{(m)} \| p_{1}^{(m)}\right)$ times the matrix in (38) is positive semi-definite because both of its leading principal minors are non-negative. Indeed, the first leading principal minor $\frac{1}{\alpha}+\frac{1}{1-\alpha}$ is obviously positive since $\alpha \in(0,1)$. The second leading principal minor is

$$
\begin{aligned}
& \left(\frac{1}{\alpha}+\frac{1}{1-\alpha}\right)\left(\frac{\alpha}{\beta^{2}}+\frac{1-\alpha}{(1-\beta)^{2}}\right)-\left(\frac{1}{1-\beta}+\frac{1}{\beta}\right)^{2} \\
= & \frac{[(1-\alpha) \beta-\alpha(1-\beta)]^{2}}{\alpha(1-\alpha) \beta^{2}(1-\beta)^{2}} \geq 0 .
\end{aligned}
$$

Since $\operatorname{ASN}_{0}^{(m)}$ is convex for all $m, \max _{m} \mathrm{ASN}_{0}^{(m)}$ is also convex. Thus, (P3) is a convex optimization problem; and hence, it can be solved efficiently.

\section{B. Operation of Sequential Sensing}

Once the optimal $\left\{\alpha^{(m)}\right\}$ and $\left\{\beta^{(m)}\right\}$ have been found, the sensing operation can proceed as follows. Collect samples until an SPRT on any band $m$ declares $\mathcal{H}_{0}^{(m)}$. Check whether the rate exceeds the required rate $R_{\min }$. If not, continue collecting samples until any of the SPRTs on the remaining bands declares absence of PUs. If the accumulated rate exceeds $R_{\min }$, stop. The number of samples collected so far corresponds to the sensing delay. Otherwise, keep sensing until all the SPRTs stop or until a prescribed maximum number $N_{\max }$ of samples have been observed. If the accumulated rate is still less than $\mathrm{R}_{\text {min }}$, declare an outage.

It should be noted that if the requested rate $R_{\min }$ is much smaller than the raw achievable rate $\bar{R}$, the sequential sensing procedure will usually terminate much earlier than the time needed for all the SPRTs to stop. In fact, in most practical scenarios, the sensing will terminate as soon as a few idle bands have been identified. Thus, the overall sensing delay will be determined by the few bands with detection delays shorter than those of the remaining bands. It is this opportunistic mechanism that leads to significant savings in sensing delay for the sequential sensing algorithm.

However, precisely because the sequential sensing stops as soon as the requested rate is deemed supportable, and because such a decision is made based on imperfect detector outputs, the sequential sensing algorithm suffers from what we term "false successes." False successes occur when the SPRTs make miss detection errors, and thus the set of bands identified as idle by the sensing procedure actually contains busy bands. Since a band $m$ occupied by a PU cannot really support rate $R_{0}^{(m)}$ for CR transmissions, the actual rate supportable by the set of bands identified by sequential sensing with the busy ones excluded, may well be less than $R_{\text {min }}$.

Note that a false success is not as problematic in FSS sensing because the detection results for all $M$ bands are available simultaneously. Although miss detection errors lead to reporting more idle bands than required by the outage constraint, the outage constraint (11) strictly enforces a set 
of truly idle bands to be identified with probability exceeding $(1-\epsilon)$; cf. (10). Fortunately, a simple remedy is possible for false successes in sequential sensing, which is elaborated next.

\section{Remedy for False Successes}

Clearly, in order to compensate for miss detection, the sequential sensing algorithm must not stop immediately after $R_{\min }$ becomes supportable. Instead, it is proposed to stop when $\mathrm{R}_{\min }+\Delta R$ is supportable, where $\Delta R>0$ denotes a rate margin introduced to counter false successes. The following lemma justifies this approach concretely.

Lemma 2. For a constant $\epsilon_{0} \in(0,1)$, and a rate margin $\Delta R \geq 0$, the following two constraints imply that the outage constraint in (11) is met:

$$
\begin{aligned}
& \operatorname{Pr}\left\{\sum_{m=1}^{M} R_{0}^{(m)} \mathbb{1}_{\left\{\mathcal{H}_{1}^{(m)} \text { true and } \mathcal{H}_{0}^{(m)} \text { detected }\right\}}>\Delta R\right\} \leq \epsilon_{0} \\
& \operatorname{Pr}\left\{\sum_{m=1}^{M} R_{0}^{(m)} \mathbb{1}_{\left\{\mathcal{H}_{0}^{(m)} \text { detected }\right\}} \leq \mathrm{R}_{\min }+\Delta R\right\} \leq \epsilon\left(1-\epsilon_{0}\right) .
\end{aligned}
$$

Proof: Consider the following (in)equalities

$$
\begin{aligned}
& \operatorname{Pr}\left\{\sum_{m=1}^{M} R_{0}^{(m)} \mathbb{1}_{\left\{\mathcal{H}_{0}^{(m)} \text { detected }\right\}} \leq \mathrm{R}_{\text {min }}+\Delta R\right\} \\
& =\operatorname{Pr}\left\{\sum_{m=1}^{M} R_{0}^{(m)} \mathbb{1}_{\left\{\mathcal{H}_{0}^{(m)} \text { true and } \mathcal{H}_{0}^{(m)} \text { detected }\right\}}\right. \\
& \left.+\sum_{m=1}^{M} R_{0}^{(m)} \mathbb{1}_{\left\{\mathcal{H}_{1}^{(m)} \text { true and } \mathcal{H}_{0}^{(m)} \text { detected }\right\}} \leq \mathrm{R}_{\min }+\Delta R\right\} \\
& \geq \operatorname{Pr}\left\{\sum_{m=1}^{M} R_{0}^{(m)} \mathbb{1}_{\left\{\mathcal{H}_{0}^{(m)} \text { true and } \mathcal{H}_{0}^{(m)} \text { detected }\right\}} \leq \mathrm{R}_{\min }\right\} \\
& \cdot \operatorname{Pr}\left\{\sum_{m=1}^{M} R_{0}^{(m)} \mathbb{1}_{\left\{\mathcal{H}_{1}^{(m)} \text { true and } \mathcal{H}_{0}^{(m)} \text { detected }\right\}} \leq \Delta R\right\} \\
& \geq \operatorname{Pr}\left\{R_{M} \leq \mathrm{R}_{\min }\right\} \cdot\left(1-\epsilon_{0}\right)
\end{aligned}
$$

where (43) is obtained because the two events inside $\operatorname{Pr}\{\cdot\}$ in (43) are independent, and jointly imply the event inside $\operatorname{Pr}\{\cdot\}$ in (42); and (44) is obtained from the definition of $R_{M}$ and (40). Upon combining (44) with (41), the desired result is obtained.

Given the design parameter $\epsilon_{0},(40)$ provides the means to calculate the margin $\Delta R$. In the operation of sequential sensing, one can stop sensing as soon as the accumulated rate based on the detector outputs exceeds $R_{\min }+\Delta R$. Then, including (41) as a constraint in the optimization formulation, the desired upper bound on the outage probability is enforced.

First, to obtain $\Delta R$ given $\epsilon_{0}$, an approach similar to that used in Lemma 1 is employed. Based on the central limit theorem, $\sum_{m=1}^{M} R_{0}^{(m)} \mathbb{1}_{\left\{\mathcal{H}_{1}^{(m)} \text { true and } \mathcal{H}_{0}^{(m)} \text { detected }\right\}}$ is treated as Gaussian with mean $\sum_{m=1}^{M} R_{0}^{(m)} \pi_{1}^{(m)} \beta^{(m)}$, and standard deviation $s_{1} \triangleq \sqrt{\sum_{m=1}^{M} R_{0}^{(m)^{2}} \pi_{1}^{(m)} \beta^{(m)}\left(1-\pi_{1}^{(m)} \beta^{(m)}\right)}$, for sufficiently large $M$. Thus, (40) can be approximated as

$$
Q\left(\frac{\Delta R-\sum_{m=1}^{M} R_{0}^{(m)} \pi_{1}^{(m)} \beta^{(m)}}{s_{1}}\right) \leq \epsilon_{0}
$$

which yields

$$
\Delta R \geq Q^{-1}\left(\epsilon_{0}\right) s_{1}+\sum_{m=1}^{M} R_{0}^{(m)} \pi_{1}^{(m)} \beta^{(m)} .
$$

Since $\Delta R \geq 0$, it follows that

$$
\epsilon_{0} \leq Q\left(-\frac{\sum_{m=1}^{M} R_{0}^{(m)} \pi_{1}^{(m)} \beta^{(m)}}{s_{1}}\right) .
$$

Similarly, $\quad \sum_{m=1}^{M} R_{0}^{(m)} \mathbb{1}_{\left\{\mathcal{H}_{0}^{(m)} \text { detected }\right\}}$ is asymptotically Gaussian distributed for large $M$ with mean $\sum_{m=1}^{M} R_{0}^{(m)}\left[\pi_{0}^{(m)}\left(1-\alpha^{(m)}\right)+\pi_{1}^{(m)} \beta^{(m)}\right]$, and standard deviation

$$
\begin{aligned}
s_{2} \triangleq & \left\{\sum _ { m = 1 } ^ { M } R _ { 0 } ^ { ( m ) ^ { 2 } } \left[\pi_{0}^{(m)}\left(1-\alpha^{(m)}\right)+\pi_{1}^{(m)} \beta^{(m)}\right.\right. \\
& \left.\left.-\left(\pi_{0}^{(m)}\left(1-\alpha^{(m)}\right)+\pi_{1}^{(m)} \beta^{(m)}\right)^{2}\right]\right\}^{\frac{1}{2}} .
\end{aligned}
$$

Then, (41) can be approximated as

$$
Q\left(\frac{\sum_{m=1}^{M} R_{0}^{(m)}\left[\pi_{0}^{(m)}\left(1-\alpha^{(m)}\right)+\pi_{1}^{(m)} \beta^{(m)}\right]-\left(\mathrm{R}_{\min }+\Delta R\right)}{s_{2}}\right)
$$

Setting $\Delta R$ equal to the lower-bound in (46), and substituting back into (49) yields

$$
\begin{aligned}
\sum_{m=1}^{M} R_{0}^{(m)} \pi_{0}^{(m)} \alpha^{(m)} \leq & \sum_{m=1}^{M} R_{0}^{(m)} \pi_{0}^{(m)}-\mathrm{R}_{\min }-Q^{-1}\left(\epsilon_{0}\right) s_{1} \\
& -Q^{-1}\left(\epsilon\left(1-\epsilon_{0}\right)\right) s_{2} .
\end{aligned}
$$

Since typically $\pi_{1}^{(m)} \bar{\beta}^{(m)} \ll \frac{1}{2}$ holds, we obtain $s_{1} \leq$ $\bar{s}_{1} \triangleq \sqrt{\sum_{m=1}^{M} R_{0}^{(m)^{2}}\left[\pi_{1}^{(m)} \bar{\beta}^{(m)}-\left(\pi_{1}^{(m)} \bar{\beta}^{(m)}\right)^{2}\right]}$. Similarly, it can be shown that [cf. (20)] $s_{2} \leq \bar{s}_{2} \triangleq$ $\sqrt{\sum_{m=1}^{M} R_{0}^{(m)^{2}} f\left(\pi_{0}^{(m)}+\pi_{1}^{(m)} \bar{\beta}^{(m)}\right)}$. Thus, a conservative surrogate for (50) is given by

$$
\sum_{m=1}^{M} R_{0}^{(m)} \pi_{0}^{(m)} \alpha^{(m)} \leq C_{3}\left(\epsilon, \epsilon_{0}\right)
$$

where

$$
C_{3}\left(\epsilon, \epsilon_{0}\right) \triangleq \bar{R}-\mathrm{R}_{\min }-Q^{-1}\left(\epsilon_{0}\right) \bar{s}_{1}-Q^{-1}\left(\epsilon\left(1-\epsilon_{0}\right)\right) \bar{s}_{2} .
$$

Thus, (P3) is solved with $C(\epsilon)$ set to $C_{3}\left(\epsilon, \epsilon_{0}\right)$ to ensure (41). In the same way as for $C_{1}(\epsilon)$ and $C_{2}(\epsilon)$, it is noted that $C_{3}\left(\epsilon, \epsilon_{0}\right)>0$ is necessary for the feasibility of condition (37). In particular, this yields a bound on $R_{\min }$ as

$$
\mathrm{R}_{\min }<\bar{R}-Q^{-1}\left(\epsilon_{0}\right) \bar{s}_{1}-Q^{-1}\left(\epsilon\left(1-\epsilon_{0}\right)\right) \bar{s}_{2} .
$$

The issue of selecting the value of $\epsilon_{0}$ will be discussed in Sec. V. 
TABLE I

OUTAGE PROBABILITIES. WHEN THE REMEDY FOR FALSE SUCCESSES IS APPLIED THROUGH $C(\epsilon)=C_{3}\left(\epsilon, \epsilon_{0}\right)$, THE OUTAGE TARGET IS STRICTLY OBSERVED.

\begin{tabular}{|c|c|c|c|c|c|c|}
\hline SNR (dB) & -7 & -6 & -5 & -4 & -3 & -2 \\
\hline \hline Prescribed $(\epsilon)$ & 0.01 & 0.01 & 0.01 & 0.01 & 0.01 & 0.01 \\
\hline Achieved $\left(C(\epsilon):=C_{1}(\epsilon)\right.$, FSS $)$ & 0.0006 & 0.0008 & 0.0005 & 0.0006 & 0.0005 & 0.0007 \\
Achieved $\left(C(\epsilon):=C_{1}(\epsilon)\right.$, seq.) & 0.0064 & 0.0070 & 0.0067 & 0.0067 & 0.0055 & 0.0058 \\
\hline Achieved $\left(C(\epsilon):=C_{2}(\epsilon)\right.$, FSS $)$ & 0.0092 & 0.0088 & 0.0098 & 0.0093 & 0.0083 & 0.0088 \\
Achieved $\left(C(\epsilon):=C_{2}(\epsilon)\right.$, seq.) & 0.0147 & 0.0138 & 0.0121 & 0.0113 & 0.0111 & 0.0099 \\
\hline Achieved $\left(C(\epsilon):=C_{3}\left(\epsilon, \epsilon_{0}\right)\right.$, seq.) & 0.0098 & 0.0090 & 0.0090 & 0.0093 & 0.0083 & 0.0088 \\
\hline
\end{tabular}

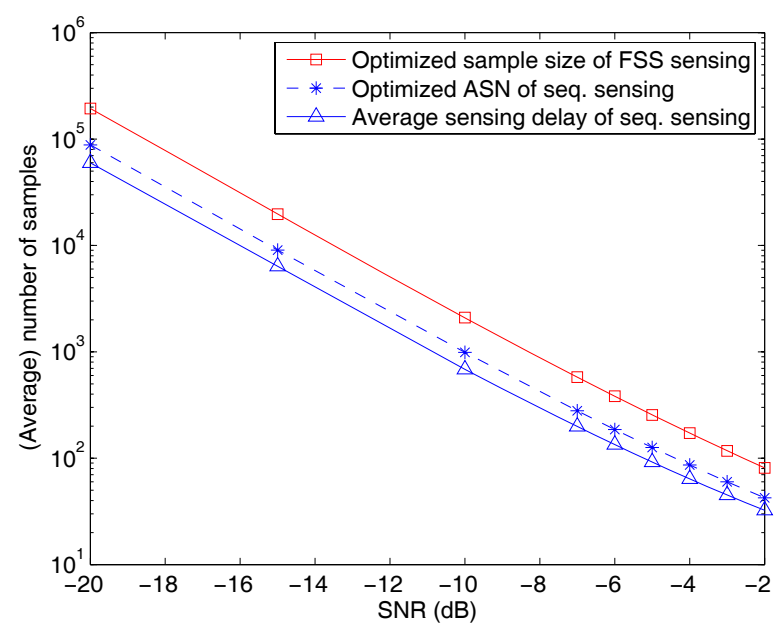

(a) $C(\epsilon):=C_{1}(\epsilon)$.

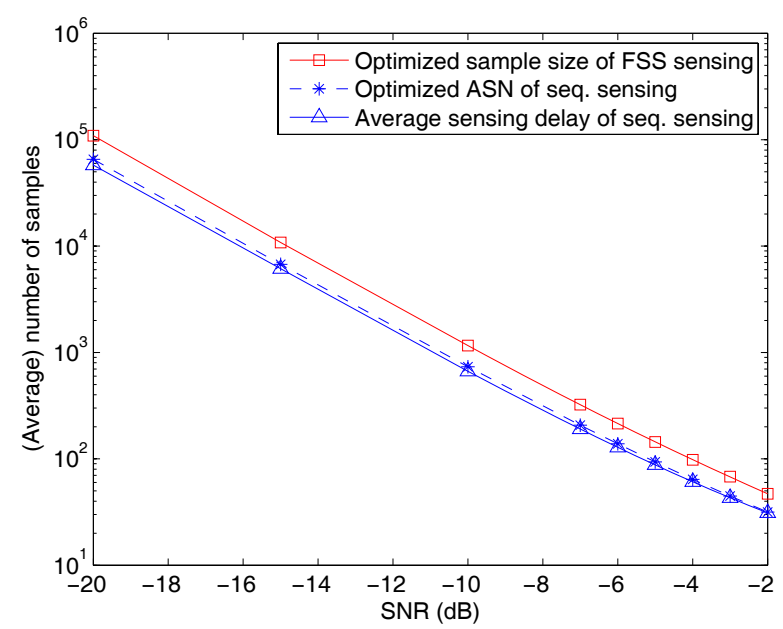

(b) $C(\epsilon):=C_{2}(\epsilon)$.

Fig. 1. (Average) sample sizes of FSS and sequential sensing. The sequential sensing saves the sensing delay significantly.

\section{Numerical Tests}

To verify the performance of the proposed FSS and sequential sensing algorithms, numerical tests are performed. A total of $M=10$ bands are available, and the achievable CR rates $\left\{R_{0}^{(m)}\right\}_{m=1}^{M}$ are given by $[2,8,10,12,14,4,6,16,18,10]$. The minimum rate requirement for $\mathrm{CR}$ traffic is given by $\mathrm{R}_{\min }=25$. The prior probability of the null hypothesis $\mathcal{H}_{0}^{(m)}$ is set to $\pi_{0}^{(m)}=1-\pi_{1}^{(m)}=0.8$ for all $m$, unless stated otherwise. The upper bounds for the false alarm and the miss detection probabilities are set to $\bar{\alpha}^{(m)}=0.4$ and $\bar{\beta}^{(m)}=0.05$, respectively, for all $m$. Note that actual false alarm rates are dictated by constraints (27) or (37). However, setting $\bar{\alpha}^{(m)}<0.5$ is necessary to ensure convexity of (P2). The number of PUs present is $J=3$, and $\bar{\beta}_{j m}=0.01$ for all $m$ and $j$. The weights $\left\{w_{j m}\right\}$ are set as $w_{j 1}=w_{j 2}=0.3$, $w_{j 3}=0.4$ for $j=1 ; w_{j 4}=w_{j 5}=0.3, w_{j 6}=0.4$ for $j=2$; and $w_{j 7}=0.1, w_{j 8}=0.2, w_{j 9}=0.3, w_{j 10}=0.4$ for $j=3$, with the rest of the weights set to 0 . The outage probability bound $\epsilon$ is set to 0.01 , unless stated otherwise. In addition, the received PU signal power is assumed to be equal in all bands. $N_{\min }=1$ and $N_{\max }=\infty$ were used. The signal and noise model follows the one set forth in Sec. II.

Fig. 1 depicts the (average) sample sizes of the FSS and the sequential sensing schemes for different values of received SNRs. Fig. 1(a) represents the case where (P1) and (P3) are solved with $C(\epsilon):=C_{1}(\epsilon)$, and Fig. 1(b) with $C(\epsilon):=C_{2}(\epsilon)$. The solid curves with square markers represent the minimized sample sizes for the FSS sensing. The dashed curves marked by stars correspond to the optimized ASN values, which are the optimal objective values of (P3). The optimized ASN value is different from the actual sensing delay since the sensing procedure stops as soon as the required rate $R_{\min }$ can be supported. The average sensing delays obtained from 50,000 independent realizations are shown as the solid curves marked by triangles. It can be seen from Fig. 1 that the sensing delay of the sequential algorithm is much lower than that of the FSS sensing. The delay reduction is more pronounced in the low SNR regime (note the log scale in the y-axis), which is the typical operating condition for CRs.

It should be noted that two avenues for delay reduction are being exploited. One is the saving achieved by opportunistic stopping of the detection procedure, which is inherent to SPRTs. This is manifested by the lower optimized ASN values compared to the sample sizes of the FSS alternatives. On top of that, the multi-band sensing architecture provides a diversity effect that further reduces delay by means of opportunistically terminating the sensing procedure whenever the minimum required rate has been achieved. The final sensing delays depicted by the solid curves with triangle markers are seen to be even lower than the optimal ASN values due to this latter effect.

Comparing Figs. 1(a) and (b), one can deduce that using $C_{2}(\epsilon)$ yields sensing delays far lower than using $C_{1}(\epsilon)$, especially for the FSS case. The outage probabilities achieved by the two approaches are shown in Table I, where it can be seen that both approaches meet the prescribed outage upper-bound in the case of FSS sensing. Therefore, using $C(\epsilon):=C_{2}(\epsilon)$ is preferred, and the remaining numerical results for FSS sensing 


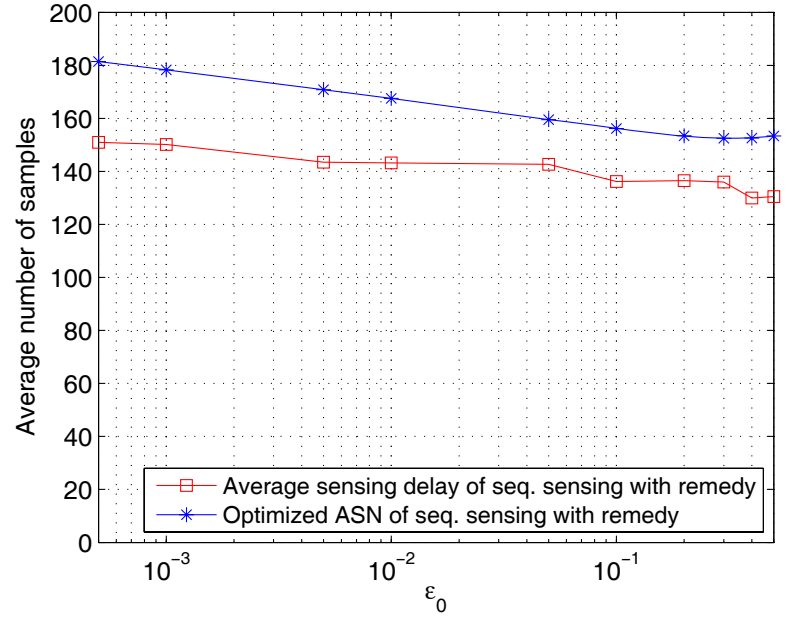

Fig. 2. Performance of sequential sensing versus $\epsilon_{0}$ at SNR $-6 \mathrm{~dB}$.

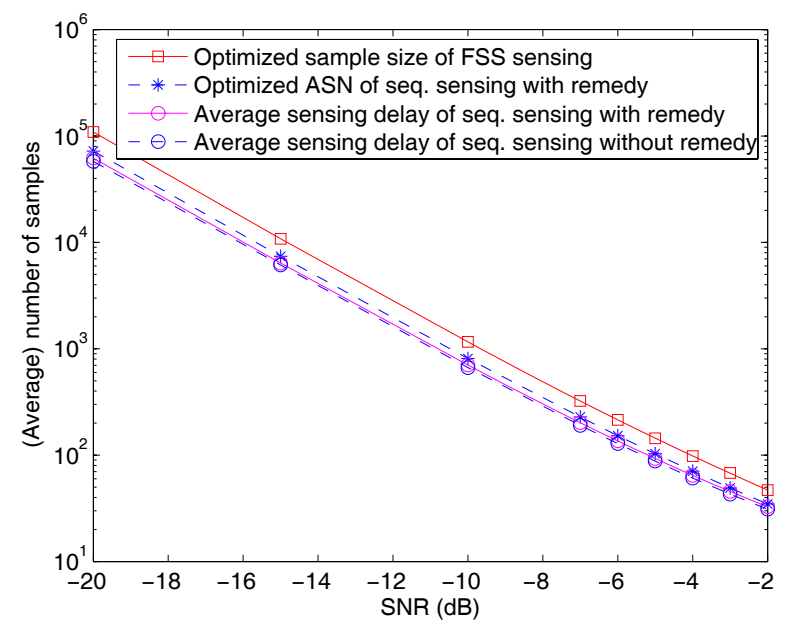

Fig. 3. Average sensing delay of sequential sensing with remedy for false successes. The remedy slightly increases the sensing delay for sequential sensing.

will be based on this choice.

In case of sequential sensing, however, Table I shows that the outage upper-bound is actually violated if $C(\epsilon):=C_{2}(\epsilon)$ is used. This is due to the false successes. To test the efficacy of the remedy for false successes proposed in Sec. IV-C, an outage constraint with $C(\epsilon):=C_{3}\left(\epsilon, \epsilon_{0}\right)$ is considered. For an appropriate choice of the value of $\epsilon_{0}$, we advocate the value that maximizes $C_{3}\left(\epsilon, \epsilon_{0}\right)$. Since $C_{3}\left(\epsilon, \epsilon_{0}\right)$ is strictly concave w.r.t. $\epsilon_{0}$, it is straightforward to find the unique maximizer, which is equal to 0.3 for the given simulation parameters. There are two justifications for this choice. First, large $C_{3}\left(\epsilon, \epsilon_{0}\right)$ renders the outage constraint (37) in (P3) less tight, so that the optimal ASN value becomes smaller. Although a small optimal ASN does not guarantee the overall sensing delay to be small, it is a reasonable choice, especially considering the lack of an analytic formula for the overall sensing delay. Secondly, as can be seen from (53), the proposed choice of $\epsilon_{0}$ maximally broadens the range of the admissible $R_{\min }$ values.

Fig. 2 depicts the optimized ASNs and the simulated

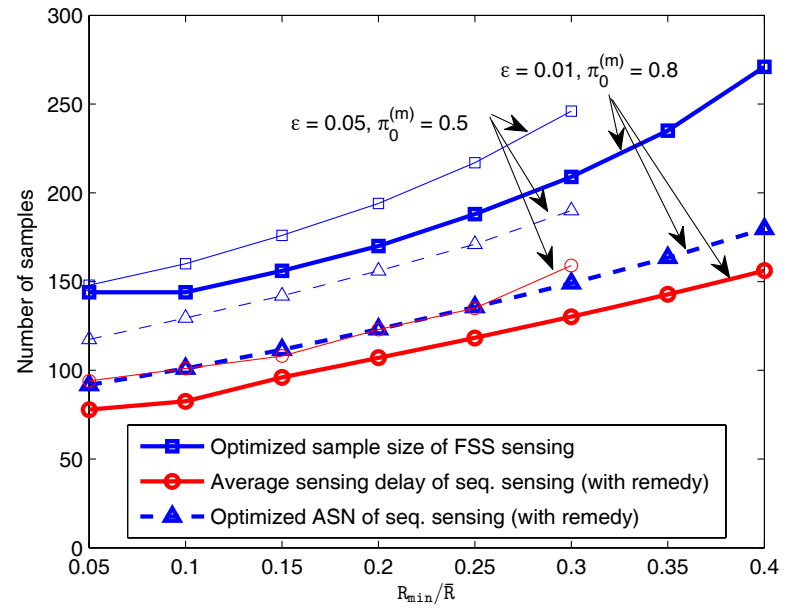

Fig. 4. Sensing delay versus $\mathrm{R}_{\min } / \bar{R}$ at $\mathrm{SNR}-6 \mathrm{~dB}$ when $\epsilon=0.01$ and $\pi_{0}^{(m)}=0.8$, as well as when $\epsilon=0.05$ and $\pi_{0}^{(m)}=0.5$.

sensing delays for different values of $\epsilon_{0}$ for $\mathrm{SNR}-6 \mathrm{~dB}$. It is verified that the smallest ASN is achieved when $\epsilon_{0}=0.3$. Although the actual sensing delay slightly decreases when $\epsilon_{0}>0.3$, the gain is negligible. In fact, per (46), too large a value for $\epsilon_{0}$ may yield a $\Delta R$ that is too small, or even negative, which may raise issues with the applicability of Lemma 2. When $\epsilon_{0}=0.3$, the corresponding $\Delta R$ turns out to be 1.16 . The outage probability obtained when the sensing procedure was terminated only when an accumulated rate of $\mathrm{R}_{\text {min }}+\Delta R$ was achieved, is shown in the last row of Table I. It is verified that the outage constraints are strictly satisfied with this approach.

Fig. 3 depicts the optimal ASNs along with the average sensing delays obtained from simulations for the sequential sensing algorithm with the remedy for false successes. Compared to the curves in Fig. 1(b), the ASNs and the average sensing delays are increased slightly to cope with the false successes. Still though, the average sensing delay is considerably lower than that of the FSS counterpart, particularly in the low SNR regime.

Fig. 4 plots the sensing delay performance at SNR $-6 \mathrm{~dB}$ when $R_{\min }$ is varied, when $\epsilon=0.01$ and $\pi_{0}^{(m)}=0.8$, as well as when $\epsilon=0.05$ and $\pi_{0}^{(m)}=0.5$. It is noted that the proposed sequential sensing algorithm is most advantageous over the FSS sensing when $R_{\min }$ is small compared to the maximum average achievable rate $\bar{R}$. Since over-provisioning of resources is often pursued to support QoS traffic with high quality-of-service, the proposed sequential sensing approach is highly attractive.

\section{CONCLUSIONS}

CR spectrum sensing algorithms that scan multiple bands in parallel to support QoS traffic were considered. Both FSS and parallel SPRT-based sequential sensing approaches were investigated. The objective was to minimize sensing delay while observing interference constraints for PU protection as well as outage probability constraints to support a requested rate for QoS traffic with high reliability. For FSS sensing, 
a convex feasibility optimization problem was formulated for a given sample size, whereby the minimum sample size satisfying all the constraints can be searched for. For sequential sensing, a convex optimization problem to minimize the maximum ASN of the parallel SPRTs was formulated so that the random stopping times of SPRTs could be approximately aligned. Subsequently, an opportunistic sensing procedure was developed that stops sensing as soon as a set of unoccupied bands capable of supporting the requested rate is obtained. Since such a stopping decision is based on imperfect detector outputs, the problem of false successes emerged. To remedy this problem, the stopping criterion was strengthened to include a rate margin, which was derived analytically to ensure the prescribed outage constraint. Numerical tests verified the efficacy of the proposed design, and demonstrated that significant reduction in sensing delay is possible through sequential sensing, especially when the operating SNR is low, and the spectral resource is over-provisioned. Future research directions include adoption of truncated SPRT [7, p. 76] to enforce a maximum sample size in sequential sensing and extensions to multi-CR cooperation [13].

\section{REFERENCES}

[1] I. F. Akyildiz, W.-Y. Lee, M. C. Vuran, and S. Mohanty, "NeXt generation/dynamic spectrum access/cognitive radio wireless networks: a survey," Comput. Netw., vol. 50, no. 13, pp. 2127-2159, Sep. 2006.

[2] S. Boyd and L. Vandenberghe, Convex Optimization. Cambridge University Press, 2004.

[3] D. Cabric, A. Tkachenko, and R. W. Brodersen, "Spectrum sensing measurements of pilot, energy, and collaborative detection," in Proc. IEEE MILCOM, Oct. 2006, pp. 1-7.

[4] S. Chaudhari, V. Koivunen, and H. V. Poor, "Autocorrelation-based decentralized sequential detection of OFDM signals in cognitive radios," IEEE Trans. Signal Process., vol. 57, no. 7, pp. 2690-2700, July 2009.

[5] R. Fan and H. Jiang, "Optimal multi-channel cooperative sensing in cognitive radio networks," IEEE Trans. Wireless Commun., vol. 9, no. 3, pp. 1128-1138, Mar. 2010.

[6] W. Feller, An Introduction to Probability and Its Applications, Vol. 2, 2nd edition. John Wiley \& Sons, 1966.

[7] Z. Govindarajulu, Sequential Statistical Procedures. Academic Press, 1975.

[8] W. Hoeffding, "Probability inequalities for sums of bounded random variables," J. Amer. Stat. Assoc., vol. 58, no. 301, pp. 13-30, Mar. 1963.

[9] J. Jia, Q. Zhang, and X. Shen, "HC-MAC: a hardware-constrained cognitive MAC for efficient spectrum management," IEEE J. Sel. Areas Commun., vol. 26, no. 1, pp. 106-117, Jan. 2008.

[10] S. M. Kay, Fundamentals of Statistical Signal Processing: Detection Theory, Vol. 2. Prentice Hall, 1998.

[11] S.-J. Kim and G. B. Giannakis, "Sequential and cooperative sensing for multi-channel cognitive radios," IEEE Trans. Signal Process., vol. 58, no. 8, pp. 4239-4253, Aug. 2010.

[12] Y.-C. Liang, Y. Zeng, E. C. Y. Peh, and T. Hoang, "Sensing-throughput tradeoff for cognitive radio networks," IEEE Trans. Wireless Commun., vol. 7, no. 4, pp. 1326-1337, Apr. 2008.

[13] S. Maleki, A. Pandharipande, and G. Leus, "Energy-efficient distributed spectrum sensing with convex optimization," in Proc. 3rd IEEE CAMSAP Conf., Dec. 2009, pp. 396-399.

[14] J. Mitola III and G. Q. Maguire, Jr., "Cognitive radio: making software radios more personal," IEEE Pers. Commun., vol. 6, no. 4, pp. 13-18, Aug. 1999.

[15] M. Öner and F. Jondral, "On the extraction of the channel allocation information in spectrum pooling systems," IEEE J. Sel. Areas Commun., vol. 25, no. 3, pp. 558-565, Apr. 2007.

[16] J. M. Peha, "Approaches to spectrum sharing," IEEE Commun. Mag., vol. 43, no. 2, pp. 10-12, Feb. 2005.

[17] H. V. Poor and O. Hadjiliadis, Quickest Detection. Cambridge University Press, 2009.
[18] Z. Quan, S. Cui, A. H. Sayed, and H. V. Poor, "Optimal multiband joint detection for spectrum sensing in cognitive radio networks," IEEE Trans. Signal Process., vol. 57, no. 3, pp. 1128-1140, Mar. 2009.

[19] H. Su and X. Zhang, "Cross-layer based opportunistic MAC protocols for QoS provisioning over cognitive radio wireless networks," IEEE J. Sel. Areas Commun., vol. 26, no. 1, pp. 118-129, Jan. 2008.

[20] Z. Tian and G. B. Giannakis, "Compressed sensing for wideband cognitive radios," in Proc. Int'l. Conf. Acoustics, Speech Signal Process., vol. 4, Apr. 2007, pp. 1357-1360.

[21] P. Wang, D. Niyato, and H. Jiang, "Voice-service capacity analysis for cognitive radio networks," IEEE Trans. Veh. Technol., vol. 59, no. 4, pp. 1779-1790, May 2010.

[22] Y. Wu and D. H. K. Tsang, "Distributed power allocation algorithm for spectrum sharing cognitive radio networks with QoS guarantee," in Proc. IEEE INFOCOM Conf., Apr. 2009, pp. 981-989.

[23] Y. Xin and H. Zhang, "A simple sequential spectrum sensing scheme for cognitive radio," IEEE Trans. Signal Process., May 2009, submitted. Available: http://arxiv.org/abs/0905.4684.

[24] Q. Zhao and J. Ye, "When to quit for a new job: quickest detection of spectrum opportunities in multiple channels," in Proc. IEEE MILCOM, Nov. 2008, pp. 1-6.

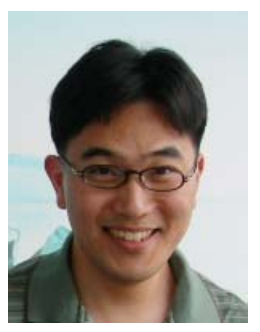

Seung-Jun Kim received his B.S. and M.S. degrees from Seoul National University in Seoul, Korea in 1996 and 1998, respectively, and his Ph.D. from the University of California at Santa Barbara in 2005, all in electrical engineering. From 2005 to 2008, he worked for NEC Laboratories America in Princeton, New Jersey, as a research staff member. Since 2008, he has been with the Department of Electrical and Computer Engineering at the University of Minnesota, where he is currently a research assistant professor. His research interests lie in applying signal processing and optimization techniques to various domains including wireless communication and networking, smart power grids, bio and social networks.

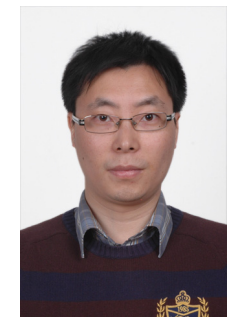

Guobing Li (S'07) is a lecturer in the School of Electronic and Information Engineering, Xi' an Jiaotong University, Xi' an, China. He received the B.S. and M.S. degree both in Electrical Engineering from Xidian University, Xi'an, China, in 2001 and 2004, respectively, and the Ph.D. degree in Electrical Engineering from Xi' an Jiaotong University in 2011. During 2008-2010 he was a visiting student at the Department of Electrical and Computer Engineering, University of Minnesota, Twin Cities. His research interests include wireless relaying, network coding and spectrum sensing in cognitive radio systems.

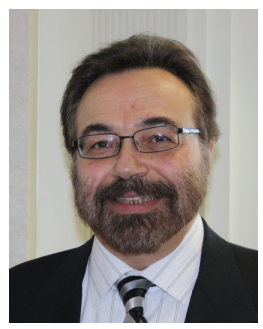

G. B. Giannakis (Fellow'97) received his Diploma in Electrical Engr. from the Ntl. Tech. Univ. of Athens, Greece, 1981. From 1982 to 1986 he was with the Univ. of Southern California (USC), where he received his MSc. in Electrical Engineering, 1983, MSc. in Mathematics, 1986, and Ph.D. in Electrical Engr., 1986. Since 1999 he has been a professor with the Univ. of Minnesota, where he now holds an ADC Chair in Wireless Telecommunications in the ECE Department, and serves as director of the Digital Technology Center.

His general interests span the areas of communications, networking and statistical signal processing - subjects on which he has published more than 300 journal papers, 500 conference papers, 20 book chapters, two edited books and two research monographs. Current research focuses on compressive sensing, cognitive radios, network coding, cross-layer designs, wireless sensors, social and power grid networks. He is the (co-) inventor of twenty patents issued, and the (co-) recipient of seven paper awards from the IEEE Signal Processing (SP) and Communications Societies, including the G. Marconi Prize Paper Award in Wireless Communications. He also received Technical Achievement Awards from the SP Society (2000), from EURASIP (2005), a Young Faculty Teaching Award, and the G. W. Taylor Award for Distinguished Research from the University of Minnesota. He is a Fellow of EURASIP, and has served the IEEE in a number of posts, including that of a Distinguished Lecturer for the IEEE-SP Society. 